\title{
Prognostic evaluation of patients with acute variceal bleeding
}

\author{
Salvador Augustin ${ }^{\mathrm{a}, *}$, Laura Millán ${ }^{\mathrm{a}}$, Antonio González ${ }^{\mathrm{a}}$, Mar Coll ${ }^{\mathrm{a}}$, María Martell ${ }^{\mathrm{a}}$ and \\ Joan Genescà ${ }^{a}, b$ \\ ${ }^{a}$ Liver Unit - Department of Internal Medicine, Hospital Universitari Vall d'Hebron, Institut de Recerca (VHIR), \\ Universitat Autònoma de Barcelona, Barcelona, Spain \\ ${ }^{\mathrm{b}}$ Centro de Investigación Biomédica en Red de Enfermedades Hepáticas y Digestivas (CIBEREHD), Instituto de \\ Salud Carlos III, Madrid, Spain
}

\section{Introduction}

In the present paper, we will review the available evidence on the prognosis of the acute variceal hemorrhage. The aim of this review is to provide some clues that may help to understand why none of the available predictive models in this setting is routinely used in clinical practice, and to identify new ways to improve our current prognostic tools. For this purpose, we will cover first some theoretical aspects that may serve as a framework to evaluate a prognostic model. In the second part of the paper, specific issues about the current knowledge of the prognosis of the acute bleeding will be revised.

\section{Prognostic tools and clinical utility}

Prognostic models are commonly regarded as more or less complex tools for providing clinicians with 3 different valuable uses: they can help physicians take difficult clinical decisions (such as ordering aggressive therapies in selected patients) [1]; select uniform groups for clinical trials [2]; or inform patients and their entourage about the odds of different outcomes in a specific situation [3]. Therefore, prognostic models are most likely to be useful for clinicians in situa-

\footnotetext{
* Corresponding author: Salvador Augustin, M.D. Liver Unit-Department of Internal Medicine. Hospital Universitari Vall d'Hebron. Passeig Vall d'Hebron 119-129, 08035 - Barcelona, Spain. Tel.: +34 932746140; E-mail: saugustin@vhebron.net.
}

tions in which "decision making is complex, when the clinical stakes are high or when there are opportunities to achieve cost savings without compromising patient care" [4]. The clinical scenario of an acute variceal bleeding clearly meets these criteria. However, none of available models in acute variceal bleeding is routinely used, and currently all patients receive the same treatment, regardless of their baseline estimated risk of complications.

The reasons for this situation are diverse. Several authors [5,6] have suggested different frameworks to evaluate the value of a given prognostic model (or clinical decision rule) to try to understand why most will not found a place in patient care. The characteristics that make a prognostic model clinically useful have been described by some authors [7] as relevance, validity and work to access. Besides, a prognostic tool that meets these criteria should proof its utility in a specific clinical context, helping doctors to inform specific clinical decisions. Finally, even if a prognostic model is valid, relevant, easy to use and informed by the clinical context, it may not be accepted by clinicians at the point of care for a variety of reasons. We will examine all these features in turn (see summary in Table 1).

\subsection{Validity}

For a model to be accepted by clinicians, it should have proven to be accurate in predicting the outcome of interest. The prognostic rule should rarely fail to predict an event that will occur (low false negative rate) and seldom predict it when it will not occur (low false 
Table 1

Desirable features of a clinically useful prognostic model (Adapted from Ebell) [6]

\begin{tabular}{ll}
\hline 1. Validity & $\begin{array}{l}\text { The model should have been proven its accuracy in predicting the outcome, ideally in a large test } \\
\text { set independent from the cohort from which the model was derived } \\
\text { The model should address a clinical important (severe and/or frequent) outcome and its use should } \\
\text { directly help improving patient care, not just diagnostic or prognostic accuracy }\end{array}$ \\
2. Relevance & $\begin{array}{l}\text { It should be easy for clinicians to obtain all necessary data and to calculate the model's prediction } \\
\text { for an individual patient } \\
\text { The design of the model should be based on the clinical scenario in which it will be eventually used } \\
\text { in daily practice } \\
\text { The model has been proven to be valid, is readily applied at the point of care, its logical structure } \\
\text { is apparent for the non-statistician and its predictions make sense to the clinicians who will rely on } \\
\text { them }\end{array}$ \\
\hline
\end{tabular}

positive rate). This would allow the prognostic model to have a desirable impact on patient's care in terms of safety (no high-risk patients would be spared from necessary interventions) and efficiency (no low-risk patients would be selected to undergo unnecessary procedures). Moreover, these error rates should be checked on a large independent test set, ideally in different time and place, and using widely accepted inclusion criteria and definitions. This might help convincing clinicians that model predictions derived from a specific cohort can be safely and efficiently used in their own patients. For the case of acute variceal bleeding, very few studies have proven their validity and generality using this standards [8-10].

\subsection{Relevance}

To be relevant for a physician, the prognostic model should be able to predict an outcome that is clinically important in terms of severity and/or frequency of the condition. Besides, in order to gain acceptance from practitioners, the model should demonstrate that its use is able to improve patient-oriented outcomes (e.g., survival, time free of severe complications - such as rebleeding, for instance- or symptoms) and/or the use of available resources in a real-world situation, than merely allowing accurate predictions. This sort of studies, which would indeed require considerable resources, are considered by some to be as necessary for prognostic models as phase 3 randomized controlled trials for drugs [11].

\subsection{Work to access}

It should be simple for clinicians to obtain all necessary data and to calculate the model's prediction for an individual patient. Those models with a small number of variables (i.e., that are parsimonious), ideally collected at patient's bedside, would be more easily ac- cepted than other models requiring more time and/or effort to acquire the needed inputs for the model. Similarly, models for which predictions take the form of printed trees [10] or clinical algorithm [12], will probably be used more often than those requiring access to computers to make complex calculations [13].

\subsection{Clinical context}

The design of a prognostic model should be based on the clinical scenario in daily practice in which it will be eventually used. To fully understand this point, a threshold model of medical decision making has been proposed, which identifies 3 actions based on the results of a decision support tool (such as a prognostic model) [14] (Fig. 1):

- identify a group of patients who need no further diagnostic/prognostic work-up because the outcome of interest is ruled out,

- identify a group of patients who need no further diagnostic/prognostic work-up because the risk of outcome is so high that empirical therapy is justified, and

- identify a group of patients who would require further evaluation because the estimated risk is not as certain as in the two previous categories and a safe decision cannot be made with the available information.

Taking the clinical context into account in the design of prognostic tools means that the choice of cutoff points for risk categories (low, intermediate, high) should be informed by these actions in the specific clinical context in which they will be used, rather than simply by maximizing statistically relevant, but clinically meaningless criteria (such as sensitivity, specificity or the area under the receiver operating characteristics curve). All too often, the latter set of criteria is favored when designing and reviewing prognostic studies, lead- 


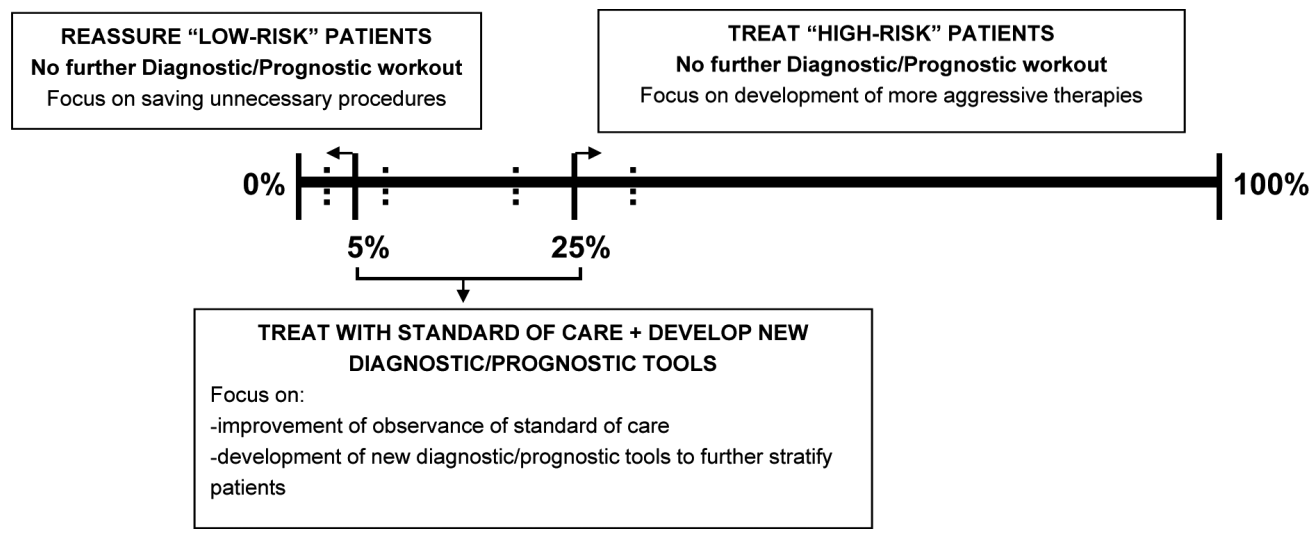

Fig. 1. Threshold model of medical decision making illustrating three different clinical and research approaches in acute variceal bleeding according to potential cut-off points of 6-week mortality based on available knowledge on the efficacy of current treatments and prognostic tools $[9,10,38]$.

ing to the publication of tools that look valid and relevant on paper, but result unhelpful in the context of real clinical decisions. If the low-risk patients have not a low-enough risk to rule out the outcome, and the high-risk patients are not at high-enough risk to justify empiric therapy, the prognostic model would be clinically useless because in every case the physician would need additional confirmatory evidence to act according to the prediction, regardless of its accuracy.

\subsection{Acceptability}

A prognostic model may have been shown to be valid, relevant, easy to use and informed by the clinical context, and yet not be accepted for practitioners. Besides hypothetical economic incentives or medico-legal concerns, which are beyond the scope of these analysis, it has been shown that physicians may reject an specific prognostic model for different reasons [15]:

- The model's structure might not be apparent and/or its predictions might not make sense to the clinicians who will rely on them. Classification trees nicely resemble the algorithmic approach to decision making [10], and would be generally favored in front of "black box" models such as artificial neural networks, which structure is obscure to the clinician [16].

- The model does not take into account all data that clinicians think are relevant in the patient situation. In this case, the parsimony that is consider desirable by the researcher in order to make the prognostic tool easy to use is regarded as a limitation by the practitioner.
- Some models require extensive interaction with computers or hand-held devices, which might be considered inappropriate in front of the patient.

- Finally, some physicians think that the use of such tools deprives the clinician of the opportunity to reason independently.

As seen, the physician's attitude towards a specific prognostic tool might be uncertain at the first stages of its design, but is critically important and must be considered when developing and updating a predictive model for clinical use.

In summary, for the case of the acute variceal hemorrhage, it should be remarked that the so-called prognostic models should not be regarded as "prognostic" in the sense of forecasting future events, as these tools are not accurate enough for this purpose. As we will see in the following sections, what these tools are wellsuited for is to retrospectively classify patients into categories or strata of individuals sharing a specific combination of clinical characteristics and comparable outcomes. In the specific context of the acute bleeding episode, this feature may be of great use for clinical and research purposes to identify target subpopulations in trials evaluating risk-based strategies.

\section{Time frame of the acute bleeding}

According to the last international consensus workshop of Baveno on portal hypertension, the time frame for the acute bleeding episode should be 120 hours (5 days) [17]. Therapeutic measures started at day 6 and thereafter are considered to be aimed at preventing the recurrence of bleeding, and are regarded as sec- 


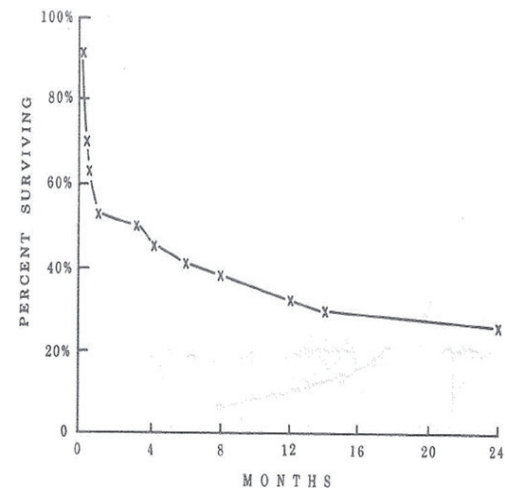

(a)

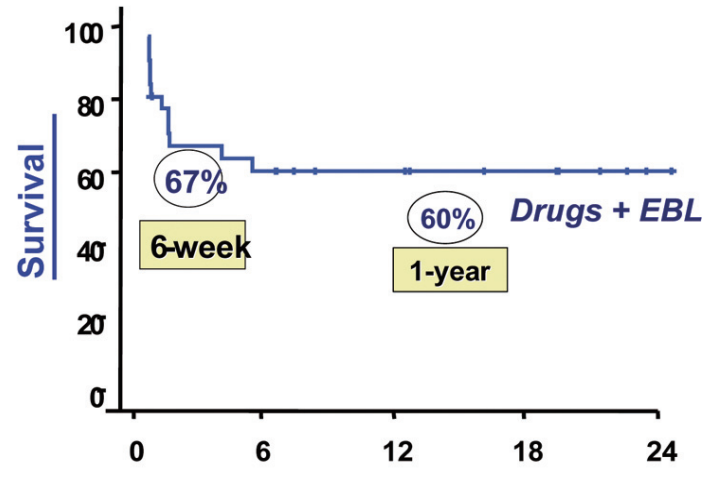

(b)

Fig. 2. Survival curve of patients after a variceal hemorrhage and clustering of mortality in the earlier 6-week period in a cohort study of untreated patients* (a), with permission [19]; and in a therapeutic study of patients randomized to early TIPS or combined pharmacologic plus endoscopic therapy (b), with permission of the author [39]. *Patients in the study did not receive specific hemostatic therapy, except for $13 \%$ of patients who received vasopressin during the acute bleeding.

ondary prophylaxis (or elective treatment) of variceal bleeding. Within this 5-day frame, the most relevant outcome by consensus has been failure to control bleeding (or treatment failure). This is a composite endpoint which includes death, persistence of uncontrolled bleeding and/or occurrence of a rebleeding episode intense enough to prompt a change of therapy. After day 5 , the clinical event that is most often considered the endpoint of interest is the occurrence of a significant rebleeding episode.

The 6-week period following the index bleeding episode is widely used in therapeutic trials and prognostic studies, although it has been only tangentially addressed in guidelines $[17,18]$. The identification of a critical 6-week period frame after an acute variceal bleeding was originally published in 1981 [19]. In their seminal paper, Graham and Smith showed that the incidence of mortality, rebleeding, and other complications, increases sharply during the first 6 weeks and then stabilize, returning afterwards to the level previous to the bleeding episode (Fig. 2a). This observation of clustering of complications in the 6 weeks following the acute episode has been constantly reproduced in many studies ever since. Even the improvement in the management of these patients, with a 4-fold reduction in mortality in the past 3 decades [20], has not altered this dynamic (Fig. 2b) [21], which can therefore be regarded as the single most robust observation in the prognosis of the acute variceal bleeding. This consistent dynamic, along with the difficulty of identifying a single cause of death after the bleeding (hemorrhage vs. liver failure vs. renal failure, etc.), has led by consensus to the pragmatical assumption that any death occurring in the 6-week period following a variceal bleeding should be considered as secondary to the acute hemorrhagic episode [22].

\section{What factors? Under what therapies?}

Although it may be seem obvious, it is worth remarking that when addressing the prognosis of an acute variceal bleeding, we are implicitly referring to the efficacy of the therapies being used. There is no point to separate this two concepts in practice. The same should be considered when comparing factors across different studies. A given prognostic factor could be relevant under a particular treatment approach, and loose its prognostic value if therapy is changed. An example could be the value as risk factor of the observation of active bleeding at the initial endoscopy, which may vary greatly on whether the patients in the study were receiving an effective vasoactive drug or not. Therefore, in order to interpret adequately the current clinical value of a prognostic factor, it is essential to take into account the treatment approach used in the studies from were its value is derived.

This evident influence of efficacy of treatments on prognosis is the main reason why the predictive value of prognostic models may change over time, and stresses the need to update our prognostic tools as therapeutic approaches are being modified. In acute variceal bleeding, this becomes especially relevant because as the efficacy of hemostatic treatments improves, the incidence of treatment failure decreases and so does the relative weight of variables strictly related to the intensity of the 
acute hemorrhage (e.g., shock, active bleeding, transfusion requirements, etc.). Consequently, it is likely that other variables (such as infection or worsening of renal failure) become more relevant.

\section{5-day failure vs. 6-week mortality}

Although considering a 5-day frame for the acute bleeding greatly simplifies the design of therapeutic trials (especially for the case of hemostatic therapies), we believe that 5-day failure may not be the most adequate endpoint to assess the prognosis after a variceal hemorrhage. The first problem encountered is the definition of failure to control bleeding, which is based on an essentially subjective and prone-to-change concept ("need to change therapy") [17]. In fact, despite the efforts made over the years to provide valid and objective criteria to generalize the indication of change of therapy, consensus is still elusive.

The second problem of the 5-day failure endpoint is that this time interval is not long enough to account for the whole spectrum of complications related to the bleeding episode, since some important complications related to the bleeding episode may require more time to develop. Therefore, some interventions (mainly hemostatic therapies) may proof to reduce 5-day failure, but may result unable to extend their impact on harder outcomes (6-week mortality) [23], which may imply an overestimation of their practical value. And inversely, other therapies able to improve 6 week survival (such antibiotic prophylaxis, for instance [24] may fail to proof their value if the analysis period is limited to 5 days. As a consequence and in analogy, the estimation of prognosis at 5 days may differ significantly from that at 6 weeks.

As an alternative, 6-week mortality shows clear advantages as endpoint of prognostic studies in acute variceal bleeding. The first obvious advantage is that mortality is strictly objective, and therefore exempt from definition problems. Secondly, it comprises a great part of treatment failures (those deaths occurring in the first 5 days). Finally, and most importantly, it does take into account the prognostic implications of relevant events that tend to reveal after day 5 (worsening of liver and/or renal function, some types of infection, etc.). For all these reasons, we consider mortality at 6 weeks as the most adequate endpoint for prognostic studies in acute variceal bleeding.

\section{Prognostic factors in acute variceal bleeding}

The value of different clinical and hemodynamic variables in determining the outcome after an acute variceal bleeding has been the matter of a number of studies in the past decade. Table 2 summarizes the largest studies ( $\mathrm{N}>100$ patients) published in the last 10 years evaluating the accuracy of invasive and clinical variables in the prediction of outcomes in variceal bleeding [8-10,25-27]. In order to interpret the relative value and potential clinical usefulness of the different studies, several considerations should be kept in mind. Regarding the treatments being used, it should be taken into account that the current recommended standard of care (vasoactive drug plus endoscopic therapy plus antibiotics) was applied only in two studies [9, 10]. Also, the use of some sort of validation of the prognostic model (in the same sample or, preferably, in an independent cohort) was lacking in half of the studies. Finally, is important to remind that none of the prognostic models proposed has been adopted for routine clinical use, as stated above.

Considering only the studies depicted in Table 2, as many as 9 variables were identified as independent markers for rebleeding or failure to control bleeding and 18 for early mortality. These figures give an average of 3 "new" variables reported in each new study. This diversity is most probably due to the heterogeneous nature of the studies: different cohorts, different treatments, different baseline hypothesis, which lead to the use of different statistical approaches and the inclusion of different set of variables in multivariate analysis, all these factors leading to diverse results. Nevertheless, a pragmatic grouping of the independent risk factors found in these studies could be made based on the outcome of interest (rebleeding or mortality), and on whether the variable informs about liver function status, the intensity of the hemorrhage, or the occurrence of complications related to the bleeding (Table 3).

\subsection{Rebleeding/treatment failure}

As seen, only in a few studies was early rebleeding considered as a separate endpoint and analyzed independently from mortality. The risk of early rebleeding has been assessed in most studies together with initial failure to control the bleeding and 5-day mortality, as the composite endpoint 5-day failure. However, the risk of 5-day failure has not been homogenously reported across studies. This is probably due to pragmatic difficulties concerning the definition of the endpoint, as 


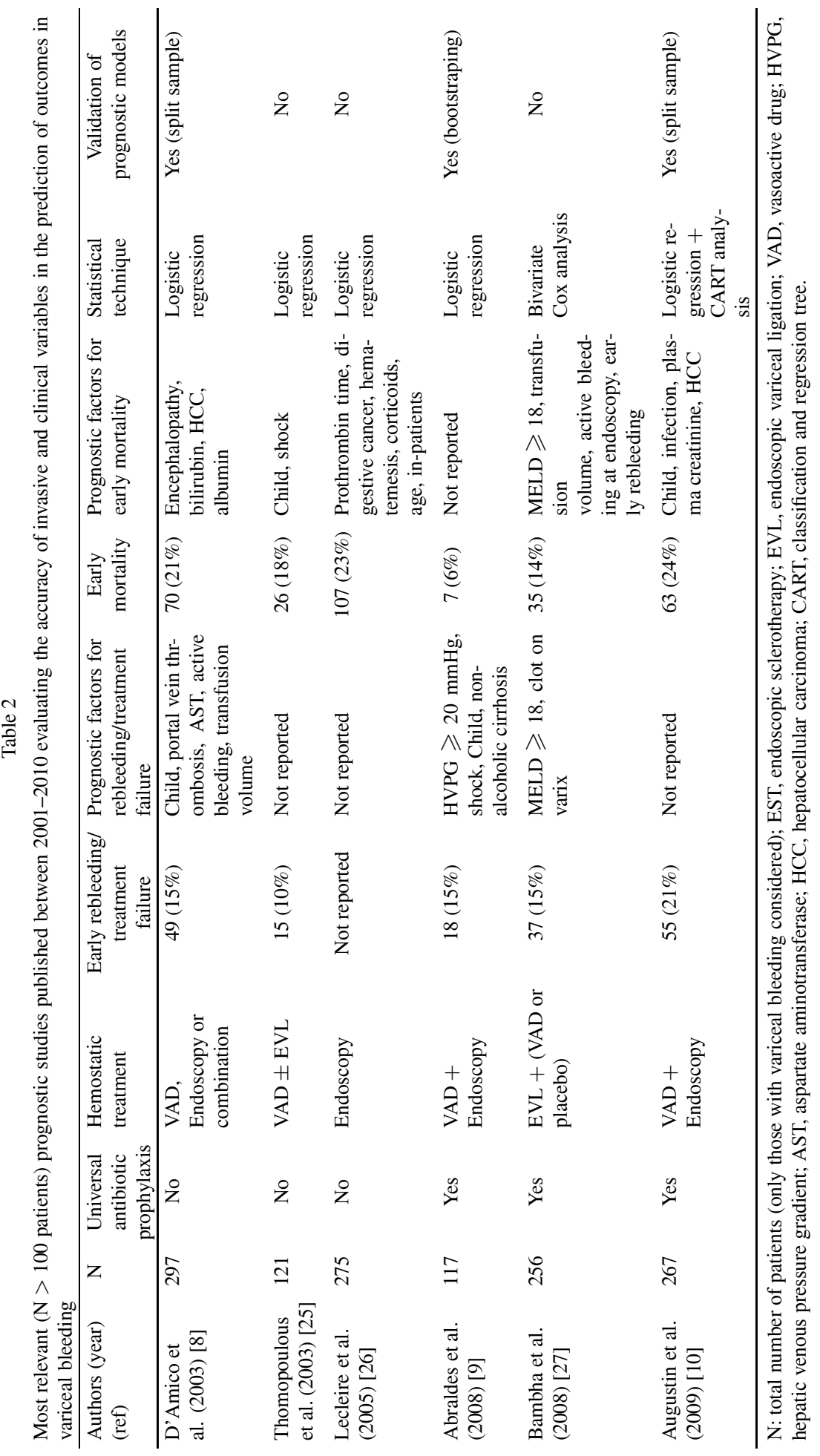


Table 3

Independent prognostic factors of rebleeding/treatment failure (A) and 6-week mortality (B) obtained from the most relevant ( $\mathrm{N}>100$ patients) prognostic studies and other therapeutic trials in acute variceal bleeding published between 2001-2010

\begin{tabular}{|c|c|c|}
\hline Rebleeding/treatment failure & Odds ratio/hazard ratio & Refs. \\
\hline \multicolumn{3}{|l|}{ Baseline liver function } \\
\hline Child-Pugh score & 1.2 & 28 \\
\hline Child-Pugh class & $17.6(\mathrm{C}$ vs $\mathrm{A}), 2.7(\mathrm{~A} / \mathrm{B} / \mathrm{C})$ & 9,8 \\
\hline MELD score & 1.04 & 27 \\
\hline Etiology of cirrhosis (non-alcohol) & 5.0 & 9 \\
\hline Portal vein thrombosis & 3.0 & 8 \\
\hline $\mathrm{HVPG} \geqslant 20 \mathrm{mmHg}$ & 5.4 & 9 \\
\hline \multicolumn{3}{|l|}{ Intensity of hemorrhage } \\
\hline Active bleeding at endoscopy & 2.1 & 8 \\
\hline Clot on varix & 2.4 & 27 \\
\hline Transfusion volume & 1.4 & 27 \\
\hline Alanine aminotransferase & 1.003 & 8 \\
\hline Hemodynamic shock & $4.9-5.5$ & 9 \\
\hline \multicolumn{3}{|l|}{ Development of Complications } \\
\hline Bacterial infection & $4.6-9.7$ & $28,29,30$ \\
\hline \multicolumn{3}{|l|}{ B) } \\
\hline 6-week mortality* & Odds ratio/hazard ratio & Refs \\
\hline \multicolumn{3}{|l|}{ Baseline liver function } \\
\hline Child-Pugh score & 1.3 & 10 \\
\hline Albumin & 0.4 & 8 \\
\hline Encephalopathy & 2.4 & 8 \\
\hline Bilirrubin & 1.2 & 8 \\
\hline Prothrombin level $<40 \%$ & 7.9 & 26 \\
\hline MELD score & 1.1 & 27 \\
\hline $\mathrm{HCC}$ & $3.1-6.0$ & 8,10 \\
\hline \multicolumn{3}{|l|}{ Intensity of hemorrhage } \\
\hline Active bleeding at endoscopy & 7.8 & 31 \\
\hline Hematemesis & 2.7 & 26 \\
\hline Transfusion volume & $1.2-1.4$ & 9,27 \\
\hline \multicolumn{3}{|l|}{ Development of Complications } \\
\hline Creatinine (baseline) & 2.3 & 10 \\
\hline Infection & 6.1 & 10 \\
\hline Early rebleeding & $3.2-8.7$ & 32,33 \\
\hline \multicolumn{3}{|l|}{ Others } \\
\hline Inpatients & 3.6 & 26 \\
\hline Digestive cancer & 4.5 & 26 \\
\hline Corticosteroids use & 3.8 & 26 \\
\hline Age $>60$ & 1.8 & 26 \\
\hline
\end{tabular}

*In Lecleire [26] the outcome was "mortality during hospitalization".

discussed above, and especially for the case of studies in which data were collected retrospectively.

Severity of the liver disease, estimated by the ChildPugh score, the Model for End-Stage Liver Disease (MELD) score or its individuals components, has been widely recognized as a robust independent determinant of 5-day failure [17,34]. A complementary set of variables reflecting the intensity of the bleeding episode (active bleeding at initial endoscopy, hemodynamic shock, transfusion requirements) has been also identified as relevant determinants of 5-day failure, as it could be expected from the definition of this outcome. The prognostic value of other variables (platelet count, etiology of cirrhosis, portal vein thrombosis) seems to be less reproducible between studies [35].

\subsection{Mortality}

When classifying patients according to their estimated risk of 6-week mortality, the baseline severity of the liver disease (mainly Child-Pugh class C) is again the main and most constant clinical determinant across studies [36]. The presence of hepatocellular carcinoma (which is most of the times closely related to liver function) has been also recognized as an important factor regarding 6-week mortality $[10,36]$. 
As previously discussed, one of the main practical implications of considering 6-week mortality as endpoint of prognostic studies in acute variceal bleeding is that the relative weight of variables reflecting the intensity of the hemorrhage decreases. Conversely, the influence on outcomes of other severe complications that may take more than 5 days to develop (such as renal failure or infections) is increased. In a recent published study by our group, development of bacterial infection and renal failure were indeed key determinants of 6-week mortality [10]. The recognition of the importance of such factors may be of great clinical value, as they are essentially potentially modifiable factors. The development of treatment strategies aimed at preventing their occurrence may have a significant impact on outcomes after an acute variceal bleeding. To this regard, the efficacy of the 7-day antibiotic prophylaxis and its beneficial impact on survival may be taken as an example [24]. Besides, the need for aggressive management of renal dysfunction in cirrhotic patients is currently widely encouraged $[18,22]$, but there are no controlled trials on the prevention or treatment of renal dysfunction after a variceal hemorrhage.

\subsection{HVPG vs. non-invasive variables and the use of TIPS in acute variceal bleeding}

The use of the hepatic venous pressure gradient (HVPG) has proven an excellent prognostic value for both treatment failure and mortality after an acute variceal hemorrhage [37]. In a multicenter trial by Monescillo et al. [31], an HVPG $\geqslant 20 \mathrm{mmHg}$ determined in the first 48 hours allowed an early classification of patients in two subpopulations with clearly different outcomes. Besides, the HVPG $20 \mathrm{mmHg}$ cutoff showed a better discriminative ability than ChildPugh score for both treatment failure and early mortality. However, it is worth remarking that the control arm in that study received only endoscopic sclerotherapy as treatment of the acute episode. This may have an influence not only in terms of the comparison of efficacy between the treatments being tested ("standard of care" vs. early TIPS insertion), but on the external validity (i.e., the reproducibility in different care settings) of the proposed prognostic rule.

In fact, in a more recent study in which all patients received combined pharmacologic plus endoscopic therapy, the prognostic ability of HVPG was compared to that of a combination of non-invasive clinical variables (Child-Pugh score, systolic blood pressure, nonalcoholic etiology) [9]. In that study, HVPG failed to improve the accuracy of the prognostic model built on clinical variables, suggesting that non-invasive variables may be sufficient to adequately stratify patients by risk in the acute episode.

In accordance with this observation, a new classification rule based on non-invasive clinical variables was used in a recent trial to stratify patients with acute variceal bleeding early after admission according to the estimated risk of treatment failure [21]. Those patients with Child-Pugh class B and active bleeding at endoscopy or Child-Pugh class $\mathrm{C}$ were considered to have a higher risk and were randomized to receive either the current standard of care or early TIPS insertion. The study showed that a treatment strategy based on early risk assessment and subsequent TIPS insertion is highly effective. An adequate selection of patients may help to optimize treatment and greatly improve outcomes in acute variceal bleeding. However, in order to assess the real value of TIPS and to optimize its use in clinical practice, two key aspects ought to be addressed. First, Child-Pugh B patients in that study had much better outcomes than Child-Pugh $\mathrm{C}$ patients (total mortality 9\% vs $42 \%$ ), suggesting that the selection rule of candidates could be improved. Second, the outcomes of patients in the control arm of that study were worse (33\% 6-week mortality) than what could be a priori expected from previous studies (8\% in patients with baseline HVPG $\geqslant 20 \mathrm{mmHg}$ ) [9]. It could be speculated that the higher mortality in the control arm of the early-TIPS study could be due in part to the fact that a proportion of patients in that group underwent sclerotherapy instead of ligation in the acute setting. To this regard, in a more recent observational study by our group, performed in a cohort of 162 patients with variceal bleeding receiving the current standard of care in the acute phase (vasoactive drugs, endoscopic ligation and antibiotics), most patients selected with the criteria used in the early-TIPS study had excellent outcomes with standard therapy (10\% 6-week mortality), suggesting that previous criteria to identify such highrisk patients may not be adequate [38,39]. Moreover, a new classification rule (Child-Pugh $\mathrm{C}$ with baseline creatinine $\geqslant 1.0$ ) to better identify a high-risk population in this setting was provided, in order to try to optimize the use of TIPS in clinical practice.

In summary, the early use of covered TIPS is highly promising and may lead to a significant change in the management of high-risk patients with acute variceal bleeding. However, the criteria used to select those high-risk patients (which are based in prognostic analyses) should be further refined as effectiveness of standard therapeutic approaches are being updated. 


\section{Conclusions}

Management of the acute variceal bleeding episode has greatly improved over the past recent years. However, treatment failures and mortality remain high, especially in patients with poor liver function, even if the current standard of care is carefully applied. Therapeutic developments and increasing knowledge in the prognosis of this complication may allow optimization of the management strategy of the acute bleeding in the near future, adapting the different treatments to the expected risk of complications for each patient. The design of accurate and manageable prognostic tools providing an early risk classification would facilitate the initiation of more aggressive treatments in highrisk patients and spare low-risk individuals unnecessary procedures. Current research efforts will hopefully clarify this hypothesis and help to further improve the outcomes of this severe complication of cirrhosis.

\section{Financial Support}

Salvador Augustin is a recipient of a 'Río Hortega' fellowship grant from the Instituto de Salud Carlos III and a 'Juan Rodés' grant from the Asociación Española para el Estudio del Hígado (AEEH). The study was partially funded by a grant from Ministerio de Ciencia e Innovación (MICINN) SAF2009-08354. CIBEREHD is supported by Instituto de Salud Carlos III.

\section{Conflict of interest disclosure}

There are no commercial affiliations or consultancies of any author that could be construed as a conflict of interest with respect to the submitted data.

\section{References}

[1] L. Holes, Loughead, T. Treasure and S. Gallivan, Which patients will not benefit from further intensive care after cardiac surgery? Lancet 344 (1994), 1200-1202.

[2] K.M. Rowan, J.H. Kerr, E. Major, K. McPherson, A. Short and M.P. Vessey, Intensive Care Society's APACHE II study in Britain and Ireland: Outcome comparisons of intensive care units after adjustment for case mix by the American APACHE II method, BMJ 307 (1993), 977-981.

[3] G. Riethmüller, E. Schneider-Gädicke, G. Schlimok, W. Schmiegel, R. Raab, K. Höffken et al., Randomised trial of monoclonal antibody for adjuvant therapy of resected Dukes' C colorectal carcinoma. German Cancer Aid 17-1A Study Group, Lancet 343 (1994), 1177-1183.
[4] T.G. McGinn, G.H. Guyatt, P.C. Wyer, C.D. Naylor, I.G. Stiell and W.S. Richardson, Users' guides to the medical literature: XXII: how to use articles about clinical decision rules. Evidence-Medicine Working Group, JAMA 284 (2000), 7984.

[5] J.C. Wyatt and D.G. Altman, Prognostic models: clinically useful or quickly forgotten? BMJ 311 (1995), 1539-1541.

[6] M. Ebell, Use of clinical decision rules for Point-of-Care Decision Support, Med Decis Making 30 (2010), 712-721.

[7] A.F. Shaughnessy, D.C. Slawson and J.H. Bennet, Becoming an information master: a guidebook to the medical information jungle, J Fam Pract 39 (1994), 489-499.

[8] G. D'Amico and R. de Franchis, Upper digestive bleeding in cirrhosis. Post-therapeutic outcome and prognostic indicators, Hepatology 38 (2003), 599-612.

[9] J.G. Abraldes, C. Villanueva, R. Banares, C. Aracil, M.V. Catalina, A.-P. Garci et al., Hepatic venous pressure gradient and prognosis in patients with acute variceal bleeding treated with pharmacologic and endoscopic therapy, J Hepatol 48 (2008), 229-236.

[10] S. Augustin, L. Muntaner, J.T. Altamirano, A. Gonzalez, E. Saperas, J. Dot et al., Predicting early mortality after acute variceal hemorrhage based on classification and regression tree analysis, Clin Gastroenterol Hepatol 7 (2009), 1347-1354.

[11] D.J. Spiegelhalter, Evaluation of medical decision-aids, with an application to a system for dyspepsia, Stat Med 2 (193?), 207-216.

[12] R.C.G. Franklin, D.J. Spiegelhalter, F. Macartney and K. Bull, Evaluation of a diagnostic algorithm for heart disease in neonates, BMJ 302 (1991), 935-939.

[13] H.A. Heathfield and I.C. Wyatt, Philosophies for the design and development of clinical decision-support systems, Methods Inf Med 32 (1993), 1-8.

[14] S.G. Pauker and J.P. Kassirer, The threshold approach to clinical decision making, N Eng J Med 302 (1980), 1109-1117.

[15] E. Toth-Pal, I. Wardh, L.E. Strender and G. Nilsson, Implementing a clinical decision-support system in practice: a qualitative analysis of influencing attitudes and characteristics among general practitioners, Inform Health Social Care 33 (2008), 39-54.

[16] A. Hart and J. Wyatt, Evaluating black boxes as medical decision-aids: issues arising from a study of neural networks, Med Inf 15 (1990), 229-236.

[17] R. De Franchis and V. the Baveno Faculty, Revising consensus in portal hypertension: report of the Baveno $\mathrm{V}$ consensus workshop on methodology of diagnosis and therapy in portal hypertension, J Hepatol 53 (2010), 762-768.

[18] G. Garcia-Tsao, A.J. Sanyal, N.D. Grace and W. Carey, Prevention and management of gastroesophageal varices and variceal hemorrhage in cirrhosis, Hepatology 46 (2007), 922938.

[19] D.Y. Graham and J.L. Smith, The course of patients after variceal hemorrhage, Gastroenterology 80 (1981), 800-809.

[20] J. Bosch, J.G. Abraldes, A. Berzigotti and J.C. Garcia-Pagan, Portal hypertension and gastrointestinal bleeding, Semin Liv Dis 28 (2008), 3-25.

[21] J.C. Garcia-Pagan, K. Caca, C. Bureau, W. Laleman, B. Appenrodt, A. Luca et al., Early use of TIPS in patients with cirrhosis and variceal bleeding, N Engl J Med 362 (2010), 2370-2379.

[22] R. de Franchis, Evolving consensus in portal hypertension. Report of the Baveno IV consensus workshop on methodology of diagnosis and therapy in portal hypertension, J Hepatol $\mathbf{4 3}$ (2005), 167-176. 
[23] C. Villanueva, M. Piqueras, C. Aracil, C. Gómez, J.M. LópezBalaguer, B. González et al., A randomized controlled trial comparing ligation and sclerotherapy as emergency endoscopic treatment added to somatostatin in acute variceal bleeding, J Hepatol 45 (2006), 560-567.

[24] B. Bernard, J.D. Grange, E.N. Khac, X. Amiot, P. Opolon and T. Poynard, Antibiotic prophylaxis for the prevention of bacterial infections in cirrhotic patients with gastrointestinal bleeding: a meta-analysis, Hepatology 29 (1999), 1655-1661.

[25] K.C. Thomopoulos, C. Labropoulou-Karatza, K.P. Mimidis, E.C. Katsakoulis, G. Iconomou and V.N. Nikolopoulou, Noninvasive predictors of the presence of large oesophageal varices in patients with cirrhosis, Dig Liver Dis 35 (2003), 473-478.

[26] S. Lecleire, F. Di Fiore, V. Merle, S. Herve, C. Duhamel, A. Rudelli et al., Acute upper gastrointestinal bleeding in patients with liver cirrhosis and in noncirrhotic patients: epidemiology and predictive factors of mortality in a prospective multicenter population-based study, J Clin Gastroenterol 39 (2005), 321327.

[27] K. Bambha, W.R. Kim, R. Pedersen, J.P. Bida, W.K. Kremers and P.S. Kamath, Predictors of early re-bleeding and mortality after acute variceal haemorrhage in patients with cirrhosis, Gut 57 (2008), 814-820.

[28] J. Goulis, A. Armonis, D. Patch, C. Sabin, L. Greenslade and A.K. Burroughs, Bacterial infection is independently associated with failure to control bleeding in cirrhotic patients with gastrointestinal hemorrhage, Hepatology 27 (1998), 12071212.

[29] B. Bernard, J.F. Cadranel, D. Valla, S. Escolano, V. Jarlier and P. Opolon, Prognostic significance of bacterial infection in bleeding cirrhotic patients: a prospective study, Gastroenterology 108 (1995), 1828-1834.

[30] S. Vivas, M. Rodriguez, M.A. Palacio, A. Linares, J.L. Alonso and L. Rodrigo, Presence of bacterial infection in bleeding cirrhotic patients is independently associated with early mortality and failure to control bleeding, Dig Dis Sci 46 (2001), 2752-2757.
[31] A. Monescillo, F. Martínez-Lagares, L. Ruiz-del-Arbol, A. Sierra, C. Guevara, E. Jiménez et al., Influence of portal hypertension and its early decompression by TIPS placement on the outcome of variceal bleeding, Hepatology 40 (2004), 793-801.

[32] Z. Ben Ari, F. Cardin, A.P. McCormick, G. Wannamethee and A.K. Burroughs, A predictive model for failure to control bleeding during acute variceal haemorrhage, J Hepatol 31 (1999), 443-450.

[33] J.A. Del Olmo, A. Pena, M.A. Serra, A.H. Wassel, A. Benages and J.M. Rodrigo, Predictors of morbidity and mortality after the first episode of upper gastrointestinal bleeding in liver cirrhosis, J Hepatol 32 (2000), 19-24.

[34] J. Bosch, A. Berzigotti, J.C. Garcia-Pagan and J.G. Abraldes, The management of portal hypertension: rational basis, available treatments and future options, J Hepatol 48 (2008), S68S92.

[35] S. Augustin, A. González and J. Genescà, Acute esophageal variceal bleeding: current strategies and new perspectives, World J Hepatol 2 (2010), 261-274.

[36] G. D'Amico, G. Garcia-Tsao and L. Pagliaro, Natural history and prognostic indicators of survival in cirrhosis: a systematic review of 118 studies, J Hepatol 44 (2006), 217-231.

[37] E. Moitinho, A. Escorsell, J.G. Bandi, J.M. Salmerón, J.C. García-Pagán, J. Rodés et al., Prognostic value of early measurements of portal pressure in acute variceal bleeding, Gastroenterology 117 (1999), 626-631.

[38] S. Augustin, J. Altamirano, A. González, J. Dot, M. AbuSuboh, F. Azpiroz et al., Effectiveness of combined pharmacologic and ligation therapy in high-risk patients with acute esophageal variceal bleeding, Am J Gastroenterol (2011), in press (doi: 10.1038/ajg.2011.173).

[39] J.C. Garcia-Pagan, K. Caca, C. Bureau, W. Laleman, B. Appenrodt, A. Luca et al., An early decision for PTFE-TIPS improves survival in high risk cirrhotic patients admitted with an acute variceal bleeding. A multicenter RCT, Hepatology 48(Suppl 1) (2008), 373A. 


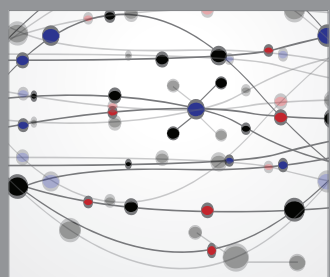

The Scientific World Journal
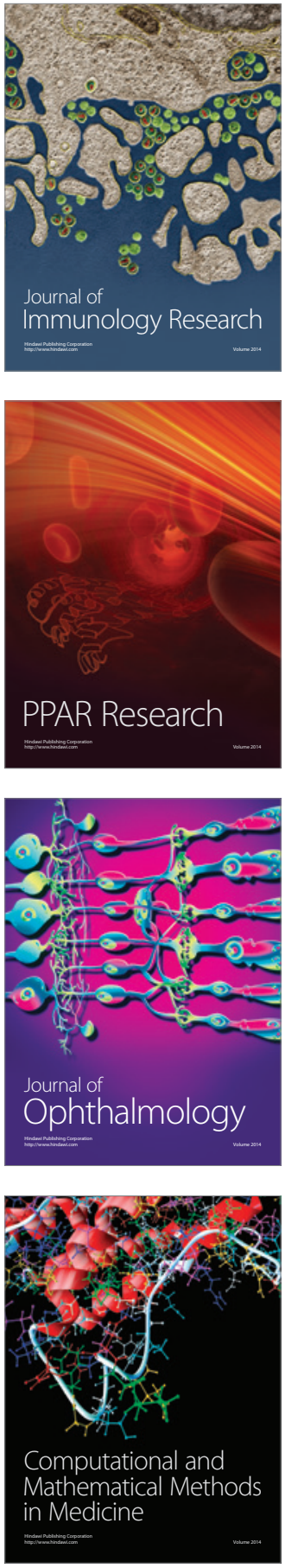

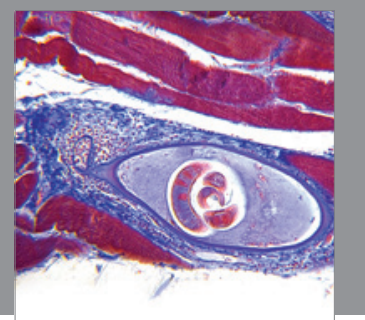

Gastroenterology

Research and Practice
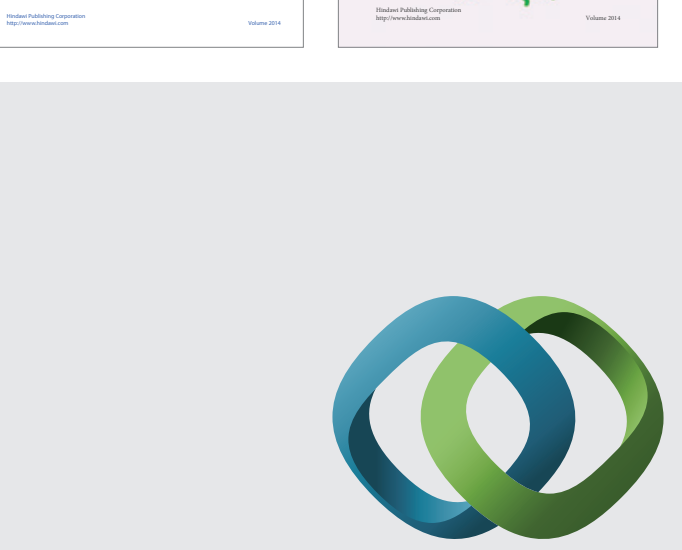

\section{Hindawi}

Submit your manuscripts at

http://www.hindawi.com
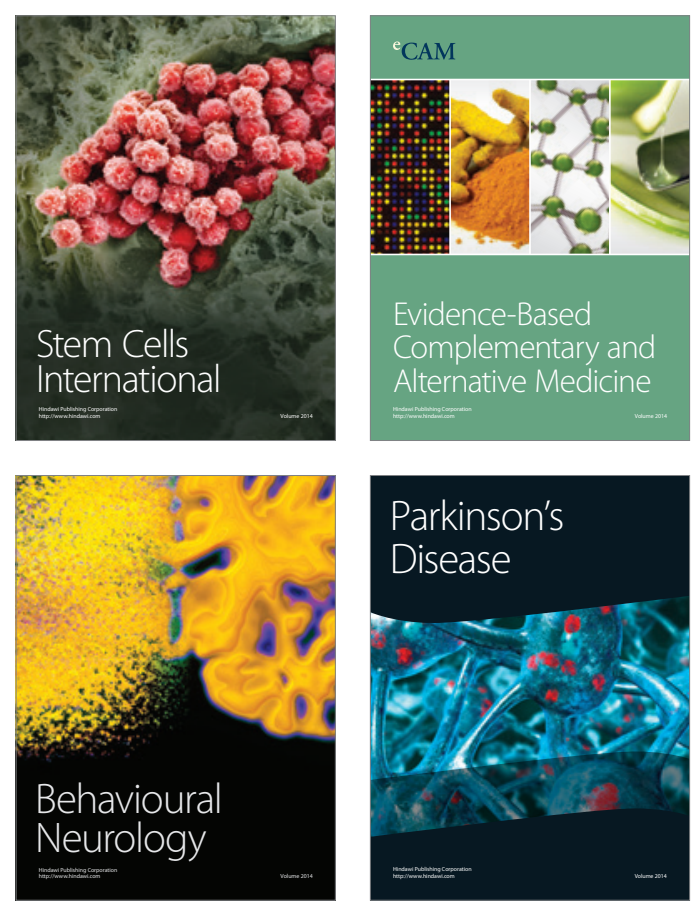

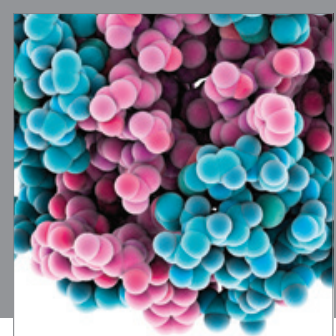

Journal of
Diabetes Research

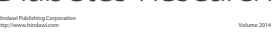

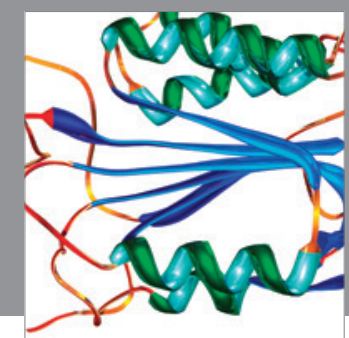

Disease Markers
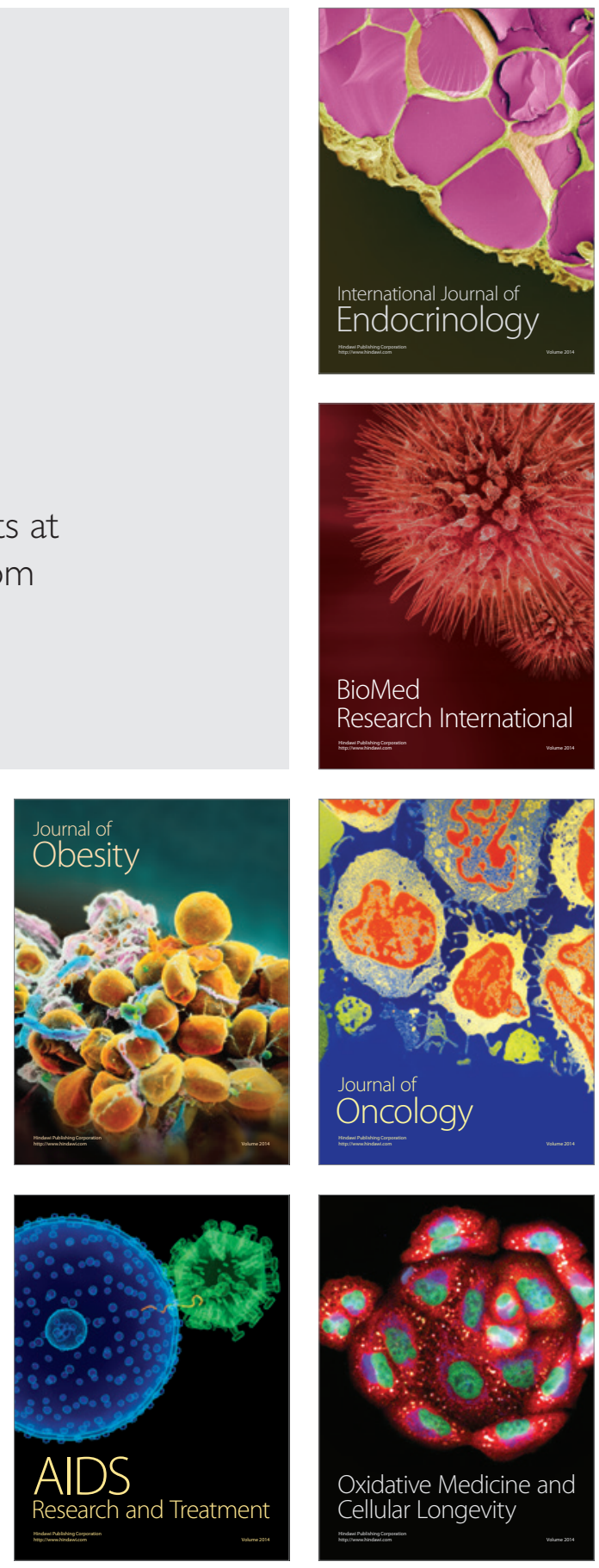